\title{
Posibilidades y límites para la inclusión social: perfiles y vías de instalación de la población dominicana en Chile
}

\section{Possibilities and limits for social inclusion: profiles and ways of installation of the Dominican population in Chile}

\author{
Caterine Galaz Valderrama \\ Co-coordinadora Académica Trabajo Social, Facultad de Ciencias Sociales, Universidad de Chile, coordinadora Núcleo Interuni- \\ versitario Estudios Críticos de la Diversidad. Doctora en Ciencias de la Educación y Magíster en Políticas Sociales. Ignacio Carrera \\ Pinto 1045.cgalazvalderrama@uchile.cl
}

\section{Gabriela Rubilar Donoso}

Centro de Estudios Interculturales e Indígenas CONICYT/FONDAP/15110006. Área Trabajo Social, Facultad de Ciencias Social de la Universidad de Chile. Doctora en Metodología de Investigación y Ciencias Sociales. Av. Capitán Ignacio Carrera Pinto 1045, Macul, Santiago.grubilar@uchile.cl

Catalina Álvarez Martínez-Conde

Núcleo Interuniversitario Estudios Críticos de la Diversidad. Psicóloga Licenciada en Psicología, Universidad de Chile.

Susana Viñuela Evans

Encargada de Contenidos y Proyectos. Fundación Alfredo Zolezzi. Licenciada en Trabajo Social. Avenida El Parque 375, oficina 203, Concón, Región de Valparaíso, Chile.susana@az-foundation.org

\begin{abstract}
Resumen
Este artículo presenta una caracterización de la situación de la población dominicana inmigrada en Chile, colectivo que no había sido foco de interés de estudios en particular y que se encuentra invisibilizado dentro de otras investigaciones que caracterizan a la población inmigrada en general. Este colectivo en Chile ha aumentado significativamente en los últimos años, según información del Departamento de Extranjería y Migraciones, la llegada de personas dominicanas se triplicó en los últimos años (DEM, 2016). Los resultados se basan en un estudio mixto, cuanticualitativo desarrollado en cuatro centros urbanos del país (Santiago, Iquique, Antofagasta y Punta Arenas). Entre los resultados cuantitativos encontrados se destaca la feminización de este colectivo, la alta concentración en un tramo de edad laboral, que se declaran en su mayoría como soltero/a con hijos/as en su país de origen, aunque sólo un 20\% vive con sus hijos/as en Chile.
\end{abstract}

Palabras clave: República Dominicana, inmigración, inclusión, feminización, derechos humanos

\footnotetext{
Abstract

This article presents a characterization of the situation of the dominican population immigrated in Chile; collective that had not been the focus of particular studies and which is therefore invisible within other studies that characterize the immigrant population in general. This group in Chile has increased significantly in recent years: according to information provided by the Department of Immigration and Migration, the arrival of Dominican people has tripled in recent years (DEM, 2016). The results presented are based on a mixed research: quantitative-qualitative study developed in four urban centers of the country (Santiago, Iquique, Antofagasta and Punta Arenas). Among the results is the feminization of this group, the high concentration in a working age segment - between 25 and 29 years old - most of them declare like a single, although most of them have children in their country of origin, and only 20\% live with their children in Chile.

Keywords: Dominican Republic, immigration, inclusion, feminization, human rights
} 


\section{Introducción y contextualización}

El exponencial incremento de movimientos poblacionales a nivel intrarregional ha ido modificando significativamente los escenarios sociales de Chile y cuestionando en ello diversas formas de comprender la inclusión social, la ciudadanía y la convivencia interna. En los últimos 30 años, el país en particular se ha vuelto un horizonte de instalación, que se ha acentuado a partir de los noventa (Tijoux y Retamales, 2015), para diversos países latinoamericanos y de Centroamérica, como es el caso de República Dominicana. Chile, de esta manera, comienza a aparecer como un horizonte posible para una instalación a largo plazo. El país, según algunos estudios, dispondría actualmente de unas 387.000 personas inmigradas, lo que correspondería a un 2,8\% de la población (DEM, 2015). Dentro de las causas de la movilidad internacional -proceso mundial definido por la creciente diversificación de flujos intra y extra regionales, una pronunciada feminización y el incremento de la migración por canales irregulares- se podrían considerar el incremento de las inversiones y el comercio a nivel mundial, el impacto de las políticas tanto de ajuste estructural como de liberalización que fueron aplicadas en los países de la región, el incremento de la pobreza, el desempleo y los niveles de informalidad que provocan mayores grados de exclusión social en algunos países, las desigualdades en los niveles de desarrollo y de oportunidades laborales y salariales entre países, la existencia de mercados que demandan mano de obra calificada y no calificada y la existencia y consolidación de redes y comunidades transnacionales (Mujica, 2004).

Comprender la situación en que se sitúan las personas inmigradas en Chile resulta vital para poder desarrollar sistemas que realmente sean de acogida y promuevan la inclusión efectiva y la participación plena de estos sectores sociales, además de poder ampliar las nociones de ciudadanía vigentes. De esta manera, este artículo presenta algunas luces sobre los perfiles del colectivo dominicano residente en Chile, considerando sus trayectorias de emigración y sus condiciones sociales de instalación, en cuatro centros urbanos del país (Antofagasta, Iquique, Santiago y Punta Arenas). Asimismo, describir sus motivaciones para su salida de República Dominicana y los vínculos que mantienen con el país de origen tanto a nivel de remesas como de contactos trasnacionales. Asimismo, se identificaron las trayectorias de ingreso que llevan a cabo, como también las condiciones de instalación en cuatro sectores prioritarios (salud, educación, vivienda y trabajo).
Cabe considerar que República Dominicana fue desde el siglo XIX un lugar de recepción de personas inmigradas, principalmente haitianas -aunque también del Caribe anglófono- alentadas por la expansión de la industria azucarera. Sin embargo, desde la crisis económica que sufrió ese país en los años 80 y la pérdida de importancia de la producción azucarera, se intensificó la salida de personas a otros países. Este país tiene un porcentaje importante de su población fuera del país, según la OCDE: cerca del 13\% de su población actualmente reside en el extranjero. Ya desde los años 80, parte de la población buscó nuevas posibilidades principalmente en Estados Unidos y Puerto Rico, España e Italia, beneficiándose -hasta antes de la crisis económica europea- de la flexibilización de visados y restricciones para la entrada. La Encuesta Comunitaria Americana sitúa la cifra de personas dominicanas en el exterior en un 1,3 millones (OCDE, 2009). En particular cabe señalar que, pese a que el modelo asumido en la década de los 80 en ese país (basado en la instauración de zonas francas de exportación y masificación del turismo) ha mejorado los índices macroeconómicos, no ha mejorado el poder adquisitivo de los/las ciudadanos/ as dominicanos/as, lo que se une a una percepción de deterioro en los servicios públicos. Todo ello ha hecho que la población dominicana busque nuevos horizontes para su desarrollo.

Morales (2007) señala que la emigración con fines laborales en el último siglo se produce en dos períodos en República Dominicana: en las décadas de 1960 y 1970, cuando la migración transfronteriza se dirigía hacia áreas de plantaciones agrícolas de difícil acceso, así como a los principales centros urbanos de las ciudades centroamericanas, teniendo a Honduras y Costa Rica como los principales destinos; y la segunda etapa (registrada a partir de la década de los años 80 e intensamente en los 90), época en la que se produjo una transformación en los flujos emigratorios no sólo en su magnitud sino también en su composición. Uno de los cambios más destacables es el aumento sustancial de la participación de las mujeres en estos movimientos, marcando una clara tendencia a la feminización de estos flujos dominicanos. Este aumento se ha sostenido a lo largo del tiempo, habiendo en los principales países de destino una amplia población femenina, la cual para el año 2010 se encontraba por sobre el $50 \%$ de la población total residiendo en esos territorios (CAREF, 2015, p. 24).

Entre el año 2002 y 2004, República Dominicana se ve como país amenazado nuevamente por una crisis 
económica, a propósito de la contracción de la economía estadounidense y la quiebra de varios bancos dominicanos, emergiendo un nuevo ciclo migratorio. En este último período se instalaría una cierta tendencia hacia la emigración irregular, fortalecido por las políticas de control migratorio ya no solo en Estados Unidos y Puerto Rico, sino también en los países europeos (Marrero y Gutiérrez, 2009). Este fenómeno, como veremos, también afecta a Chile en los últimos años, al solicitarse visado en el país de origen desde el año 2012. Según estos autores, con estas exigencias, se acrecienta el establecimiento de redes de tráfico y trata especialmente de mujeres, con fines de explotación sexual.

Por otro lado, cabe señalar que las relaciones diplomáticas y consulares entre Chile y República Dominicana se han establecido desde las primeras décadas del siglo XIX, pero no es hasta el año 2008, en el marco de las Primeras Consultas Políticas, Económicas y de Cooperación, que se establece una agenda de trabajo para el establecimiento y fortalecimiento de las relaciones bilaterales entre ambos países. Esto ha potenciado la realización de convenios y acuerdos económicos, políticos y culturales. En ese sentido, últimamente se han establecido diálogos entre ambos países con el fin de promover acuerdos comerciales (Ministerio de Relaciones Exteriores, 2015), así como el establecimiento de tratados respecto a la cooperación técnica y científica. Uno de los acuerdos más relevantes en materia migratoria que tiene incidencia directa en la instalación de la población inmigrada a Chile actualmente tiene que ver con el restablecimiento del requisito de visa de turismo para personas dominicanas con pasaporte ordinario.

Luego de que identificaran problemáticas asociadas a la trata de personas en la que fueron identificadas víctimas de nacionalidad dominicana, se llevaron a cabo intercambios de notas diplomáticas entre personeros de ambos gobiernos. Esto resultó en la decisión chilena de instalar en el año 2012 el requisito de visa de turismo para establecer una regulación de los flujos migratorios provenientes de República Dominicana. En el estatus de turista se encuentran las personas que ingresan al país con fines recreativos, deportivos, de salud, estudios, negocios, familiares, religiosos u otros similares, y que no tienen intenciones de inmigración, residencia definitiva o desarrollo de actividades remuneradas. La visación de turismo se otorga por un máximo de 90 días, y para su tramitación se establecen una serie de requisitos que deben presentarse ante el consulado chileno en República Dominicana, en- tidad encargada de revisar, aprobar o rechazar las solicitudes de visado junto al Departamento de Inmigración del Ministerio de Relaciones Exteriores de Chile (2016). Veremos que esta investigación pudo constatar que las posibilidades de instalación son diferenciadas para quienes han migrado antes de este acuerdo y quienes lo hicieron posteriormente, generando diferencias en el goce de derechos efectivos de este colectivo.

\section{Línea teórica: el enfoque de derechos humanos y nuevas visiones de ciudadanía}

El respeto a los derechos humanos se ha convertido en un lugar común: su uso excesivo ha llevado inevitablemente a la imprecisión del concepto, por lo que es necesario detenerse en su definición (Ferrer, 2006; Galaz, Frías y Poblete, 2015). Los derechos humanos pueden ser entendidos en términos del conjunto de garantías universales que cada individuo puede reclamar en tanto su condición de ser humano (Ferrer, 2006; Esperón, Dantur y Carnero, 2012). La violación a algún derecho en particular, o la inexistencia de condiciones socioeconómicas, políticas y ambientales adecuadas para su exigencia y ejercicio, implica poner en riesgo la totalidad de derechos que le otorgan a una persona su dignidad como ser humano (Gómez, 2014). A su vez, estos derechos deben ser respetados independientemente del lugar en el que la persona se encuentre, lo que pone en jaque la asociación entre adquisición de derechos, ciudadanía y nacionalidad (Muñoz, 2009).

A su vez, los derechos humanos refieren al núcleo ético del Estado y sus políticas públicas, ya que "el poder del Estado sólo se legitima sí reconoce a cada uno de los miembros de la sociedad como núcleos de capacidades de derechos y sus políticas públicas buscan respetar, proteger y satisfacer esas capacidades" (Gras, 2013, p. 8). En ese sentido, la dimensión moral de los derechos humanos expresada en su declaración necesita de una dimensión legal, en términos de política pública nacional e internacional, que permita la efectiva garantía de su ejercicio (Ferrer, 2006). Desde este lugar, la promoción y protección de los derechos humanos atraviesa distintos niveles: internacional, estatal, institucional y de la sociedad civil (Provoste, s/f), los cuales se articulan en función de garantizar el cumplimiento de los distintos derechos, independiente de los sujetos que se encuentren a su alero, siendo inaceptables las discriminaciones por raza, género, edad o clase social (Muñoz, 2009).

Esta vinculación efectiva entre distintos niveles 
implica dejar de pensar en los derechos humanos únicamente como un sistema jurídico o una propuesta ética de contenido universalista, y reconocerlos como un sistema de poder que debe ser traducido en una plataforma de políticas públicas, dotadas de herramientas de planificación, gestión y desarrollo de recursos, donde la sociedad civil también juega un importante rol en la medida en que se compromete por su respeto, exigencia y defensa (Galaz, Frías y Poblete, 2017).

Sin embargo, la relación entre Estado y Derechos Humanos se encuentra afectada por el contexto social, ya que los objetivos de la neoliberalización y del poder del mercado, en contraste con los objetivos de las políticas públicas, han generado crisis en la identidad y en los roles del Estado como garante de derechos (Gras, 2013). Las lógicas de mercado se encuentran orientadas a la maximización del bienestar individual, mientras que las políticas públicas se orientan hacia el bienestar general, siendo decisiones con una irreductible lógica política, que buscan proveer de servicios universales a todos quienes sean ciudadanos con derechos, lo que genera conflictos al momento de trazar las líneas de desarrollo de las distintas naciones. Actualmente, se ha reconocido que el desarrollo del mercado y la democracia formal no generan de por sí las condiciones estructurales para garantizar los medios de existencia y bienestar, ya que para ello es primordialmente necesario que exista un mecanismo de redistribución de las riquezas y altos niveles de participación social y política de la ciudadanía en las políticas públicas sociales (Muñoz, 2009).

Así, los Estados tienen la obligación de promover el respeto universal, la observancia y la protección de los derechos humanos, como también de atender a la relación dependiente entre democracia, desarrollo y derechos (Gras, 2013). En esta última relación, se considera que el ser humano es el sujeto central del desarrollo y que él debe ser el participante activo y beneficiario de dicho derecho. Para esto, los Estados deben implementar políticas de desarrollo nacional adecuadas para mejorar el bienestar de la población completa, independiente de su nacionalidad de origen, prestando atención a una participación activa, libre y significativa en el desarrollo y en la equitativa distribución de los beneficios resultantes de este (Vallenas, 2011).

Por otro lado, es importante considerar que las exclusiones y problemas sociales que atraviesan la migración no son estrictamente causa de la migración como fenómeno en sí mismo. Tal como señala Yufra (2013), las personas inmigradas no plantean "nuevas" problemáticas a las sociedades receptoras, sino más bien ponen de manifiesto los límites y tensiones que ya se encontraban en ellas, como es la concepción misma de ciudadanía. En ese sentido, cada sociedad receptora construye su propia migración en función del tratamiento que hace de la presencia de las personas inmigradas. El rol que cumplen las políticas de control migratorio es relevante al generar categorizaciones sociales que determinan las condiciones de vida de las personas inmigradas, por lo que muchas veces las políticas focalizadas en la integración de la población inmigrada lo que hacen es subsanar las situaciones de fragilidad y vulnerabilidad que las mismas políticas migratorias producen, influyéndose mutuamente.

Sin embargo, existen discursos que tienden a presentar las problemáticas asociadas con la instalación como un proceso unidimensional, responsabilidad únicamente de la idiosincrasia cultural del "inmigrado/a". Las sociedades que funcionan mediadas por estos discursos persiguen el ideal de la integración, el cual es definido como un proceso de "cambio cultural" individual en el que los migrantes adoptarían los valores y normas propios de la sociedad de instalación, resultando muchas veces en la invisibilidad social de la diferencia y diversidad (Santamaría, 2002, Galaz, 2009).

En este marco, las fronteras legislativas impiden el acceso a espacios fundamentales de desarrollo personal y social a personas, grupos o comunidades que no cumplen los requisitos nacionales, lo que produce que las poblaciones migrantes, junto a otros colectivos, sean excluidos de la cultura ciudadana a pesar de ser titulares de derechos universales (Pérez de la Fuente, 2006; Peña, 2012; Autora, 2006). Por esto, las migraciones internacionales tienen el efecto de restaurar la estratificación cívica en el interior de las sociedades, al establecer la barrera entre quienes son sujetos ciudadanos y quienes son residentes, pero no ciudadanos/as, encontrando en esta última categoría a los/las migrados/as regulares, ilegales, en tránsito, etc. (Peña, 2012). Estas diferenciaciones se encuentran explícitamente reglamentadas en las normas de los Estados democráticos, lo que impide que los distintos sujetos puedan disfrutar de todos los derechos que el estatus de ciudadano confiere, lo que genera una jerarquización perversa de ciudadanos de primera, segunda o tercera categoría (Muñoz, 2009; Autora, 2006). Esto también permite una diferenciación entre ciudadanos/as y no-ciudadanos/as en cuanto a las condiciones requeridas por el acceso a los servicios sociales, de los cuales los/las ciudadanos/as gozan sin necesi- 
dad de ningún requisito previo, así como también, la exclusión de los derechos políticos propios de la ciudadanía, impidiendo la autonomía política, es decir, la posibilidad de tomar parte en la decisión sobre los asuntos públicos, en especial aquellos que afectan la propia vida (Peña, 2012). Así, la asistencia a las personas inmigrantes en situación administrativa irregular queda fuera de las responsabilidades del Estado, siendo responsabilidad de otros actores generalmente ligados a la caridad y precariedad en sus prestaciones (Yufra, 2013).

Estas exclusiones, que intensifican la marginalización de los sujetos en las sociedades en las que residen (Gómez, 2014), son reforzadas por la visión de la migración como una amenaza, ya que en la libertad de ciudadanía por ejemplo, los sectores más conservadores temen la evasión de las limitaciones impuestas a la migración o la evitación de la deportación (Usher, 2004). Esto nos habla de que el sujeto inmigrado es visto como un huésped temporal, cuya presencia está condicionada a las circunstancias del mercado (De Lucas, 2004 citado en Peña, 2012). Sin embargo, la migración ha actuado como catalizador social de muchos de los cambios que está experimentando actualmente la institución de la ciudadanía, ya que como expone Javier Peña (2012, p. 529):

"[las migraciones] alteran la estructura interna, social y cultural, de las sociedades de acogida, mostrando la inadecuación del modelo vigente de ciudadanía estatal-nacional a la complejidad y diversidad de las relaciones sociales y formas de vida en las sociedades actuales, y modificando la percepción que los miembros de estas sociedades tienen sobre el significado de su condición de ciudadanos".

\section{Metodología}

El estudio se desarrolló a partir de una metodología de carácter mixto que integró tanto una perspectiva cuantitativa como cualitativa. En este artículo, se presenta la sistematización de la información cuantitativa. Los instrumentos para la recogida de información empleados en la investigación fueron preferentemente un cuestionario de caracterización, entrevistas a informantes clave y un conjunto de entrevistas semiestructuradas. El cuestionario de caracterización consistió en la aplicación de un instrumento estandarizado de preguntas cerradas que recogió información en torno a variables sociodemográficas, migratorias y de calidad de vida de la población dominicana residente en Chile, apli- cado en cuatro centros urbanos con alta presencia de personas dominicanas: Santiago, Antofagasta, Punta Arenas e Iquique. Se aplicó un total de 330 encuestas y el análisis de caracterización se realizó utilizando como herramienta de apoyo el programa SPSS.

\section{Resultados: Perfiles de las personas inmigradas dominicanas}

Las personas inmigradas dominicanas que habitan en Chile son preferentemente mujeres. Esta situación es evidenciada cuando se analizan las estadísticas del Departamento de Extranjería (2016) de los últimos 10 años (2005-2015), que reporta que el $69 \%$ de los solicitantes de visa y residencia definitiva de República Dominicana son mujeres. La encuesta de caracterización realizada sigue una tendencia similar, ya que 216 de los 330 encuestados/ as son mujeres y 114 son hombres.

\section{FIGURA N ${ }^{\circ}$ 1: PORCENTAJE MUJERES Y HOMBRES PARTICIPANTES EN EL ESTUDIO (N: 330)}

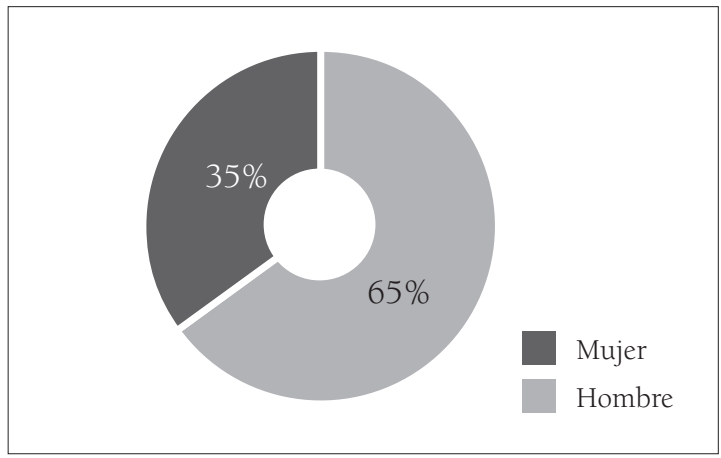

Fuente: Elaboración propia.

Al desagregar esta variable por zona de aplicación encontramos algunas diferencias: la mayor paridad de género se observa en la zona de Antofagasta, mientras que la mayor diferencia está en la ciudad de Punta Arenas, donde el 86\% (42) de las encuestadas son mujeres.

Junto con la marcada tendencia a una migración femenina, se observa que la mayor parte de los/as dominicanos/ residentes en Chile son jóvenes, que se concentran entre los 25 y 39 años (DEM, 2016). Si ordenamos a las personas encuestadas en diez tramos de edad observamos que el mayor porcentaje de éstos/as se concentra entre los 30 y 34 años (24\%) seguidos del grupo que declara tener entre 25 y 29 años (22,9\%). En consecuencia, las y los dominicanos/as que llegan a Chile se encuentran en un rango de edad laboral, es decir, adulto/a joven entre 20 y 40 años, lo que refuerza la noción de que es una inmigración de carácter laboral preferentemente. 
FIGURA N 2: EDAD DE LOS ENCUESTADOS ORDENADA POR TRAMOS (N: 330 )

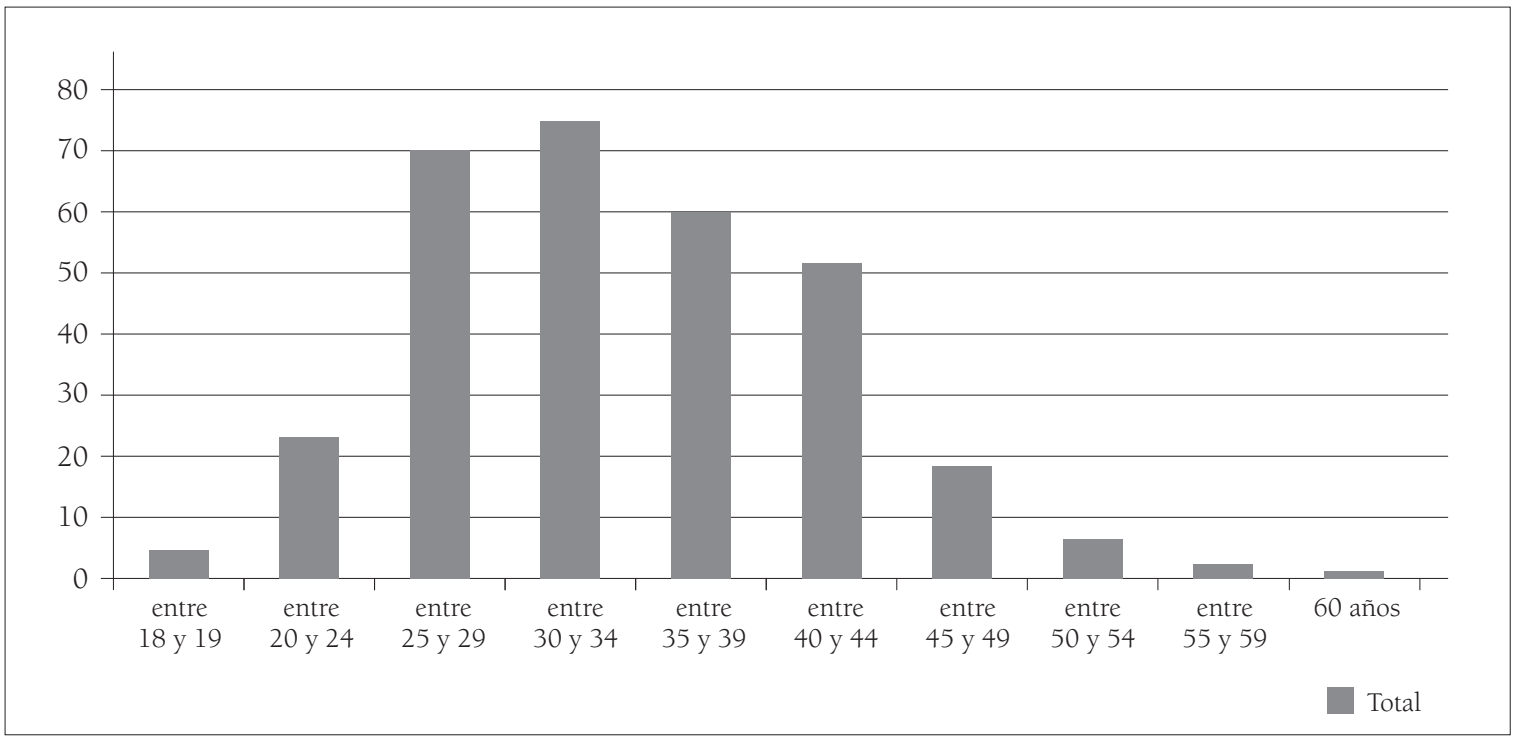

Fuente: Elaboración propia.

\section{Trayectoria migratoria}

Al llegar a Chile se instalan preferentemente en los centros urbanos asociados a las vías de ingreso, para luego desplazarse por distintas comunas o regiones en función de las oportunidades laborales. Quienes llegaron a Chile con anterioridad a la petición de visado en origen (2012) lo hicieron principalmente a través del aeropuerto internacional de la ciudad de Santiago. A través de diversas redes de solidaridad entre sus connacionales desarrollan su instalación, o bien se asentaban en espacios territoriales con alta demanda laboral en ese momento. Por tanto, sus asentamientos comenzaron a predominar en comunas donde ya residían otros/as conciudadanos/as.

Cuando se analiza la trayectoria de comunas de residencia seguidas por los/as dominicanos/as participantes en este estudio se observa que la primera comuna donde se instalan mayoritariamente es la comuna de Santiago, para desde allí desplazarse a otras comunas de las región o a otras regiones. En este estudio se observa que un 19\% de las personas encuestadas de Punta Arenas, Antofagasta e Iquique llegaron inicialmente a la comuna de Santiago, lo que da cuenta de un patrón de movimiento al interior de las distintas regiones del país con fines laborales.

De 330 encuestados/as, 241 declaran que ingresa- ron a Chile por vía aérea, lo que representa un 73\% del total de participantes de la encuesta. Como se mencionó en el punto anterior, es el aeropuerto Arturo Merino Benítez la principal vía, con excepción de dos encuestados que declaran ingresar por los aeropuertos de Chacalluta y Diego Aracena, respectivamente. Quienes ingresan por vía terrestre lo hacen principalmente por pasos fronterizos de la zona norte del país, con excepción de tres encuestados que declaran ingresar por Magallanes.

De 37 encuestados/as, 21 de Iquique declaran haber ingresado al país por pasos fronterizos del norte de Chile y sólo 6 por aeropuerto ${ }^{1}$. Los/as encuestados/ as de esta zona presentan diferencias con las vías de ingresos de los de las otras zonas, donde predomina el ingreso por aeropuerto. Un total de 17 encuestados/as no especifica la vía de ingreso al país, por lo que el análisis de esta dimensión se realiza sobre 313 casos válidos.

Del total de los/as encuestados (330), el 63\% tiene un tiempo de permanencia en el país mayor a tres años, varios de ellos/as se encuentran regularizados/ as y con visas definitivas, lo que permite una estancia en el país con mejores oportunidades. Un 36,5\% de encuestados/as lleva menos de tres años en el país, y son precisamente ellos quienes enfrentan mayores dificultades de inserción, cuando no tienen su visa-

1 Es importante consignar que 10 encuestados de Antofagasta que no responden este ítem. Por otro lado cabe consignar que un porcentaje no determinado no quiso contestar este ítem de la encuesta tanto en Antofagasta e Iquique como en Santiago. También es importante tener en cuenta que esta información contrasta con la información cualitativa, donde se destaca en los relatos que ha ido en aumento la entrada por paso fronterizo no regular. 
do de turista. El tiempo de permanencia en el país que declaran tiene directa relación con la situación migratoria de los/as dominicanos/as que residen en el país, lo que permite diferenciar distintos tipos de situaciones y distintos grados de integración a nivel social y laboral.

De esta manera se identifican tres perfiles que componen la migración dominicana: aquellas personas con ingreso regular por medio del acceso a visas consulares; aquellas con ingreso irregular por paso no habilitado luego de no haber adquirido o solicitado la visa; y aquellas que sin haber obtenido la visa en el país de origen deciden no migrar. Obviamente los registros oficiales de información refieren en su mayoría a este primer perfil, debido a que las personas con ingreso regular pasan obligatoriamente por mecanismos de control en las fronteras y en las instituciones pertinentes, como el Departamento de Extranjería. Los dos últimos perfiles de ciudadanos/ as dominicanos/as quedan excluidos/as o invisibilizados/as de los registros, pasando a formar parte de la cifra negra de la migración, mermando la ca- pacidad del Estado y de las instituciones de generar conocimiento real sobre el tipo de migración que llega o tiene intenciones de viajar desde República Dominicana a Chile, dificultando así su caracterización. Lo anterior va a dar origen a distintas formas de inserción a nivel laboral y va a determinar en parte las posibilidades de acceso a mecanismos de integración residencial y de seguridad social.

El análisis de la información obtenida en las encuestas se complementa con las percepciones de los/as entrevistados/as cualitativamente, quienes reconocen que la situación de la población dominicana ha sufrido un antes y un después, fruto del requerimiento de visa de turismo para ingresar a Chile. De este modo, la situación migratoria de las personas dominicanas se ha vuelto diferenciada para dos grandes grupos: mientras algunos/as ya poseen residencia definitiva y han podido insertarse en Chile; un porcentaje no determinado se encuentra en situación de irregularidad, enfrentando un problema de orden legal, administrativo y penal por haber ingresado al país por paso fronterizo no habilitado ${ }^{2}$.

\section{FIGURA N 3: PERFILES E IMPACTOS DE VISA DE TURISMO EN ORIGEN}

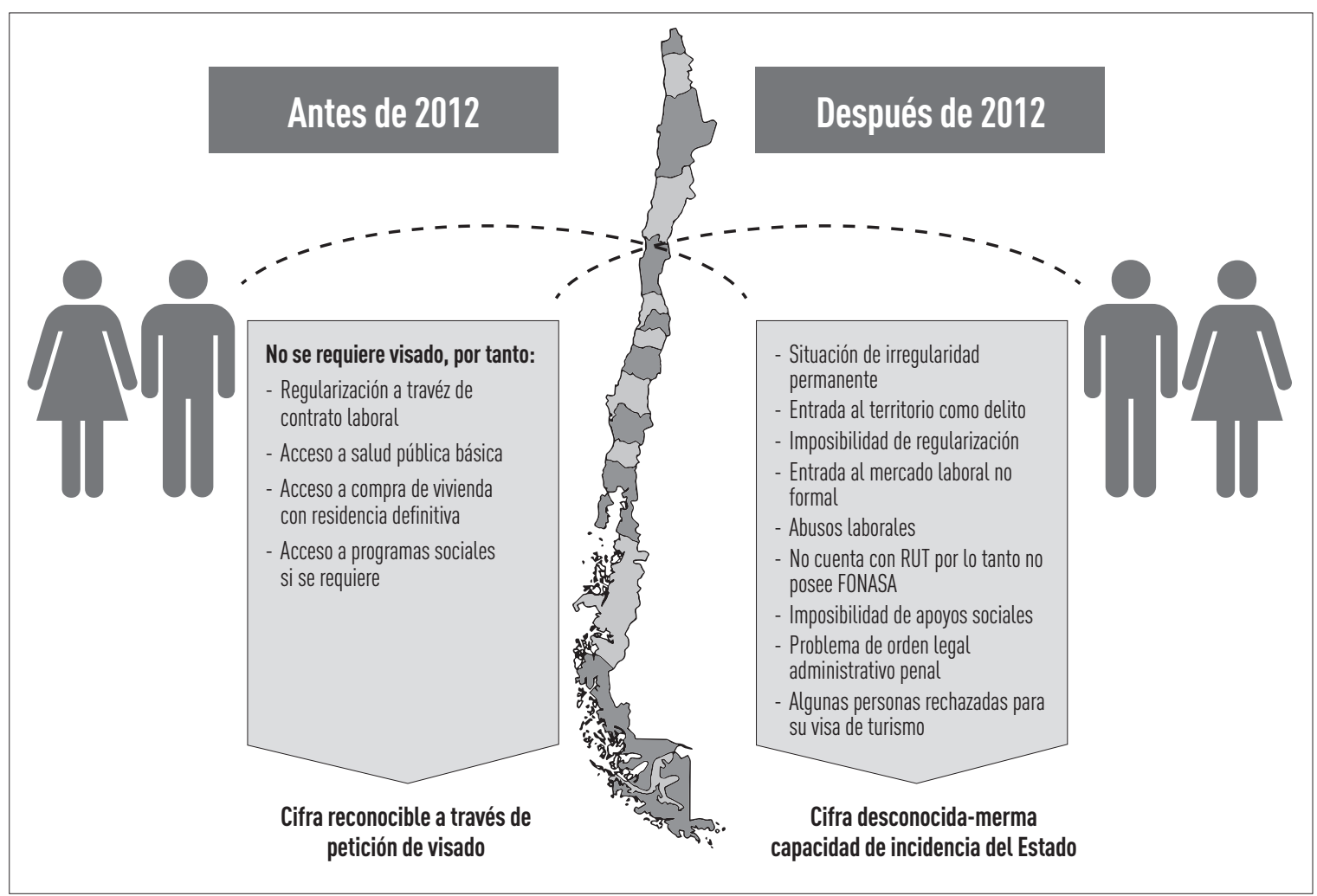

Fuente: Elaboración propia.

2 Si bien esta situación también se manifiesta en otros colectivos inmigrantes, lo importante a señalar es que la entrada al territorio por paso fronterizo no habilitado, genera una calificación de "delito" que les impide un camino formal de forma expedita para poder regularizarse. 
Quienes ingresaron al país con anterioridad al visado, lo hicieron por lo general como turistas y con el tiempo han ido regularizando su situación a través de la contratación laboral. Actualmente gran parte de los/as encuestados/as manifiesta tener visas temporales o visas definitivas, 130 de 330 encuestados/as declara poseer una visa definitiva $(39,4 \%)$, condición que se contrasta fuertemente con 83 encuestados/as que declaran no poseer visa y que se encontrarían en situación irregular (25,1\%).

La mayor parte de los sujetos encuestados que declaran poseer visa temporaria afirmó que esta visa es producto de su vínculo con chilenos/as (24 encuestados/as) o vínculo con familiar con visa definitiva (10 encuestados/as). Cuando se analiza el tipo de visa por zona de aplicación se constata que Iquique es la ciudad donde las personas encuestadas presentan mayor dificultades en su regularización, dado que el 75,7\% (28) de las personas encuestadas en esta ciudad no posee visa, ni permiso alguno.

Cuando se consulta a los/as encuestados/as, ¿cuál es la principal razón por la que no tienen visa?, la mayor parte declara que trataron de obtenerla, pero no lo lograron (29 encuestados/as), o que la obtuvieron, pero ésta se venció. Varios de ellos/as no contestan a la pregunta o dan cuenta de la enorme cantidad de documentos que deben adjuntar a su solicitud (48 encuestados/as refiere a falta de documentos o no cumplir los requisitos), lo que indica la existencia de razones principalmente económicas o administrativas que les impiden obtener esta documentación.

\section{Causas de la migración y sectores de inserción social}

Las personas inmigradas señalan que la principal razón para migrar a Chile tiene que ver con mejorar oportunidades laborales y una búsqueda por una mejor situación económica. En el caso de los/as encuestados/as predominan las respuestas en torno a la búsqueda de un trabajo $(35,8 \%)$ y una mejor situación económica $(22,1 \%)$, seguido de una motivación por una mejor calidad de vida $(21,2 \%)$. Esto respalda el carácter laboral de este tipo de migración. La mayor parte de participantes en la encuesta afirma que volverían a escoger a Chile como país para residir (202 encuestados/as lo que representa un $61,2 \%$ ), otro grupo lo escogería casi siempre $(24,2 \%)$, lo que muestra una tendencia mayorita- ria a priorizar este país como horizonte de destino. Sólo un poco más del 10\% de encuestados/as no volvería a emigrar a Chile nuevamente, mientras que otro porcentaje menor $(4,6 \%)$ piensa que lo haría casi nunca.

FIGURA N ${ }^{\circ}$ 4: PRINCIPAL RAZÓN PARA EMIGRAR (N: 330)

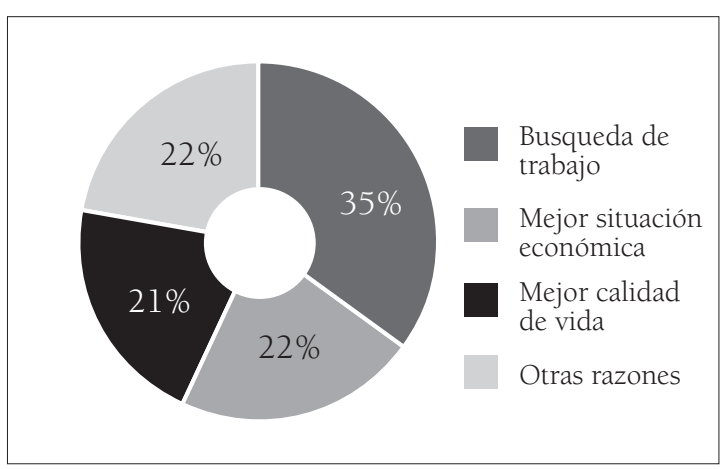

Fuente: Elaboración propia.

\section{Trabajo}

De 330 encuestados/as, 309 ha trabajado en algún momento en el país, lo que representa un 93,6\% de encuestas. El 100\% de los/as encuestados/as de Antofagasta e Iquique han trabajado alguna vez en el país, lo que presenta algunas diferencias con el Gran Santiago y Punta Arenas, donde el 7,8\% y el $10,2 \%$ de dichas zonas declaran no haber trabajado antes en Chile. Se observa que quienes declaran haber trabajado (309 encuestados/as) suelen haber tenido mayoritariamente entre 2 y 5 trabajos $(77,02 \%)$. Unos pocos entrevistados han tenido 10 trabajos o más, y la mayor parte de ellos se ubican en las zonas de Gran Santiago e Iquique ${ }^{3}$.

Sobre las redes y mecanismos usados por los/as encuestados/as para encontrar trabajo en Chile es importante consignar que el $23,6 \%$ de los/as dominicanos/as consultados/as afirma que recibió ayuda de familiares y amistades que ya trabajaban en el país, mientras que el 20,4\% buscó trabajo usando redes de amistades pero que no necesariamente estaban trabajando. Sumando ambas formas de búsqueda, nos da que el 44\% de los migrantes declara buscar empleo a través de redes primarias con personas que se encuentran en el país antes de iniciar su proyecto de emigración.

Los/as encuestados/as también dan su opinión sobre las razones o los factores que inciden en que los/

3 El $43 \%$ de quienes no trabajan afirma que no recibe aportes económicos. Otro 23\% reconoce que recibe dinero de familiares y amigos en Chile y un 28,8 recibe otros tipos de ingresos, no especificados, a partir de aportes de instituciones, del exterior u otros agentes. 
as dominicanos/as puedan ingresan al mercado del trabajo en Chile. Entre ellos, el principal factor que destacan es la disposición de ellos/as a trabajar más horas (20,9\%); mientras que la mayor dificultad es el no poseer visa de trabajo (65\%), aspecto que refuerza la importancia de los procesos de regularización para una mejor inserción laboral.

Cuando se ordena por rubro el trabajo que actualmente desarrollan se obtiene que la mayor parte de los/as dominicanos/as se concentran en dos grupos principales: comercio (27\%) y servicio doméstico (23,3\%). La construcción agrupa a un 13\% de los/ as encuestados/as, mientras que los servicios personales y de belleza declaran ser desarrollados por el 10\% de las encuestadas, que en este caso corresponde exclusivamente a mujeres. En otros rubros (27\%) se encuentran actividades relacionadas con la agricultura, el trabajo en fábricas, servicios administrativos y educacionales, entre otros

\section{FIGURA N ${ }^{\circ}$ 5: RUBROS EN LOS QUE SE DESEMPEÑAN LOS MIGRANTES ENCUESTADOS QUE TRABAJAN (N: 270)}

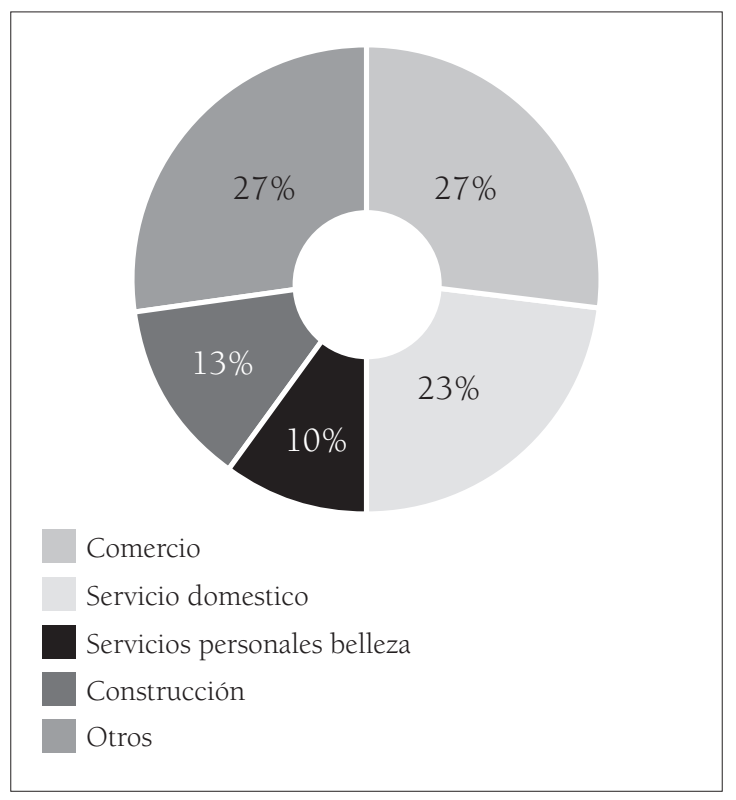

Fuente: Elaboración propia.

Al desagregar los resultados de la encuesta por zona de aplicación, se obtiene que los/as encuestados/ as de Antofagasta y Punta Arenas se agrupan en el sector comercio ( $60 \%$ y $54,1 \%$, respectivamente). En Iquique, la mayor parte de trabajadores/as se concentra en otros rubros $(40,7 \%)$ mientras que el gran Santiago predominan trabajadores/as en servicio doméstico (32,5\%), aunque esta es la zona de aplicación donde se aprecia en términos generales mayor diversidad de rubros de trabajo. Vemos que los trabajos en los que se desempeñan se encuentran claramente segmentados por género y enfatiza la feminización del proceso migratorio dominicano. Cuando los/as encuestados/as comparan su trabajo actual con el que tenían en República Dominicana, se observa que el 49,2\% de éstos/as que responden a esta pregunta califica su situación actual como "mejor" a la anterior, aspecto que es coherente con las motivaciones para migrar a Chile.

Cuando se analizan los resultados de la valoración del trabajo actual por zona de aplicación se observa que las encuestadas de Punta Arenas son quienes mayoritariamente califican su trabajo actual como peor a la de los/as encuestados/ de otras zonas (39\%). A diferencia de lo anterior, en el Gran Santiago es donde se concentra un mayor porcentaje de dominicanos/as que califica su trabajo actual como mejor al que tenía en su país de origen $(54,9 \%)$. En opinión de la mayor parte, los sueldos que perciben en Chile son "mejores" a los obtenidos por ellos en República Dominicana (77,8\%).

\section{Educación y vivienda}

El 34,5\% (114) de los/as dominicanos/as que participaron en la encuesta poseen educación secundaria completa y un 20,6\% secundaria incompleta (68). Un 15,8\% (52) de encuestados/as afirma tener estudios universitarios incompletos y un 4,5\% (15) haber completado sus estudios de carácter universitario. Porcentualmente, los niveles educativos más bajos se encuentran entre los/as encuestados/as de la zona de Punta Arenas (con mayor porcentaje de educación media completa e incompleta). También llama la atención en la zona de Antofagasta el predominio de encuestados/as con educación técnica (completa e incompleta), aspecto que está relacionado con el tipo de oficio en el cual se desempeñan los/as encuestados/as de esta zona (comercio y construcción).

Por su parte, quienes poseen educación secundaria completa mayoritariamente no han realizado las homologaciones $(93,2 \%)$ en Chile. Sólo 8 encuestados/as de este grupo afirman haberlo hecho, lo que representa un $6,8 \%$ del total de encuestados/as que se encuentran en esta situación. Finalmente entre quienes poseen estudios universitarios se constata que solo 2 encuestados/as $(11,8 \%)$ han convalidado u homologado sus estudios en el país. La mayor parte de los/as encuestados/as que poseen título universitario declaran no poseer los recursos económicos para hacer esta gestión o no poseer tiempo e información suficiente para completar los trámites 
requeridos, aspectos que se constituyen en las principales razones para no homologar los estudios.

En tanto, si bien la situación de vivienda presenta algunas diferencias por zona de aplicación, se constata que en términos generales la mayor parte de los/as encuestados/as declarar vivir en una habitación que arrienda (44\%), seguido de casa (34\%) y departamento (22\%). El 90,2\% de los/as encuestados/as arrienda la vivienda que habita. Sólo 2,8\% de los/as dominicanos/as que participa en la caracterización es propietario/a de la vivienda, lo que corresponde a 9 casos ( 7 del Gran Santiago y dos de Punta Arenas).

\section{FIGURA N ${ }^{\circ}$ : SITUACIÓN DE VIVIENDA DE LOS ENCUESTADOS (N: 329)}

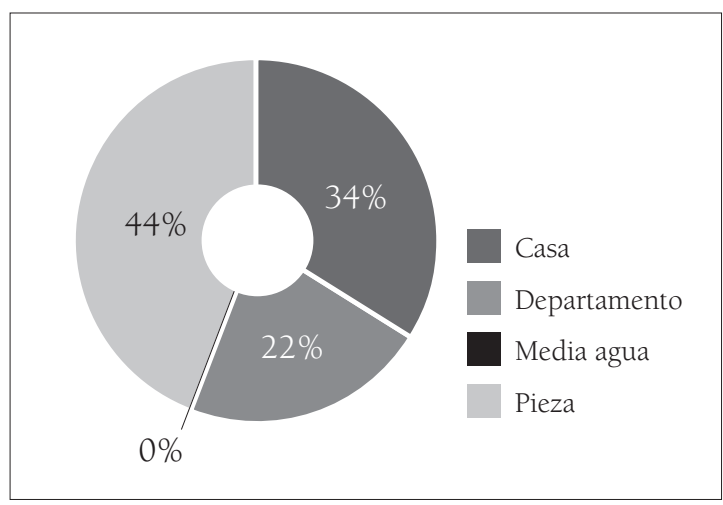

Fuente: Elaboración propia.

En tanto, más de la mitad de los/as encuestados/ as declara que vive con su familia directa $(54,9 \%)$ en la misma vivienda; seguidas de un $25,1 \%$ de las personas encuestadas que comparten vivienda con otras familias; y un 20,1\% de encuestados/as que vive solos/as.

Cuando comparan su vivienda actual con la que tenían en República Dominicana se observa que el 56,8\% de los/as encuestados/as que responden a esta pregunta califica su situación actual como "peor" a la anterior, aspecto que es coherente con las condiciones de habitabilidad que enfrentan en Chile. El resto de las respuestas se divide entre igual o mejor.

\section{Salud}

En el ámbito de salud, el 61,6\% (202) de encuestados/as afirma que ha tenido requerimiento o necesidades de salud durante el tiempo de estadía en Chile. Cuando se analizan estos resultados por zona de aplicación se observa que estas necesidades son disímiles y en buena medida están vinculadas al tiempo de permanencia de los dominicanos en el país. Los/as encuestados/as de la ciudad de Iquique son los que mayoritariamente declaran no haber requerido servicios en este ámbito (58,8\% de los encuestados): Una situación completamente distinta se observa en el caso de Antofagasta, donde la mayor parte de los/as encuestados/as afirma haber requerido atención de salud durante su estancia en el país ( $89,7 \%$ de los encuestados).

El 69,3\% de los/as encuestados/as declara que posee algún tipo de previsión de salud. Cuando se desagrega esta previsión según sistema, se observa que un 44\% cotiza en el Fondo Nacional de salud (FONASA) con algún nivel de copago, otro $18 \%$ posee gratuidad en el sistema de FONASA y un 3\% declara cotizar en isapre, porcentaje que también se repite en el caso de aquellos/as que afirman atenderse en forma particular. Un 32\% (104) de los/as participantes en encuesta afirmó que no posee seguro de salud, porcentaje que es levemente superior a aquellos encuestados/as que no poseen contrato de trabajo, y por lo mismo involucra también a otros/as dominicanos/as que no tienen cobertura de salud básica.

\section{FIGURA N ${ }^{\circ}$ 7: SISTEMA DE SALUD DE LOS ENCUESTADOS (N: 326)}

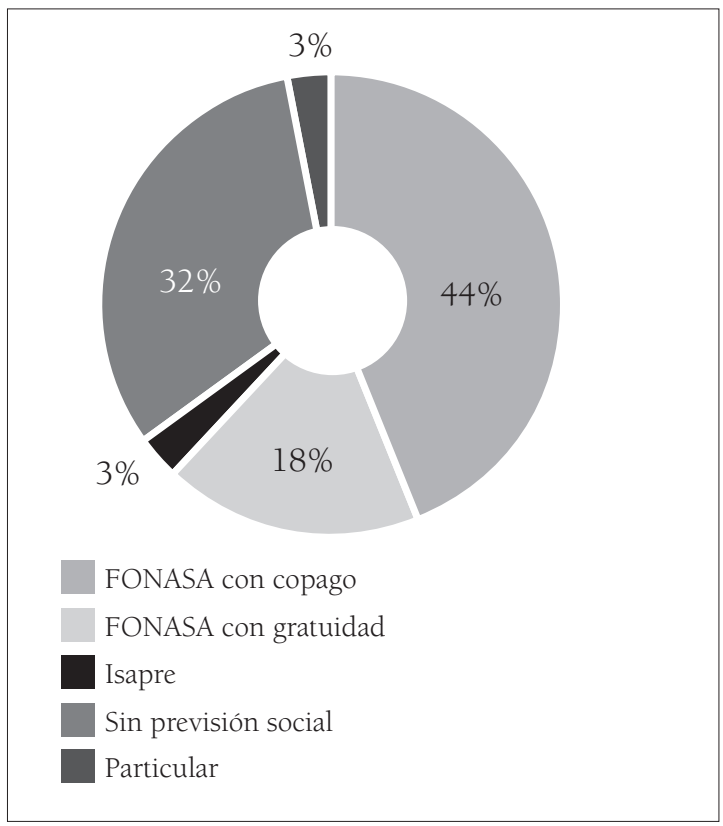

Fuente: Elaboración propia.

Cuando se analizan los datos desagregados por zona de aplicación se constata que los/as encuestados/as de Iquique son los que mayoritariamente no cuentan con ningún tipo sistema de salud $(21,1 \%$ de esa zona), seguido de los/as encuestados/as del Gran Santiago $(31,2 \%)$. 


\section{Familias, Remesas y comunicación trasnacional}

\section{Estado civil y situación de pareja}

En este estudio, 211 de los/as 330 encuestados/as se declaran solteros/as, lo que representa un $63,9 \%$ del total. Otros 68 encuestados/as conviven con sus parejas, lo que representa 20,61\% del total. En tanto, 45 encuestados/as afirman encontrarse casados/as, lo que representa el 13,64\% del total de participantes de la encuesta de caracterización.

\section{FIGURA N ${ }^{\circ}$ 8: ESTADO CIVIL DE LOS ENCUESTADOS}

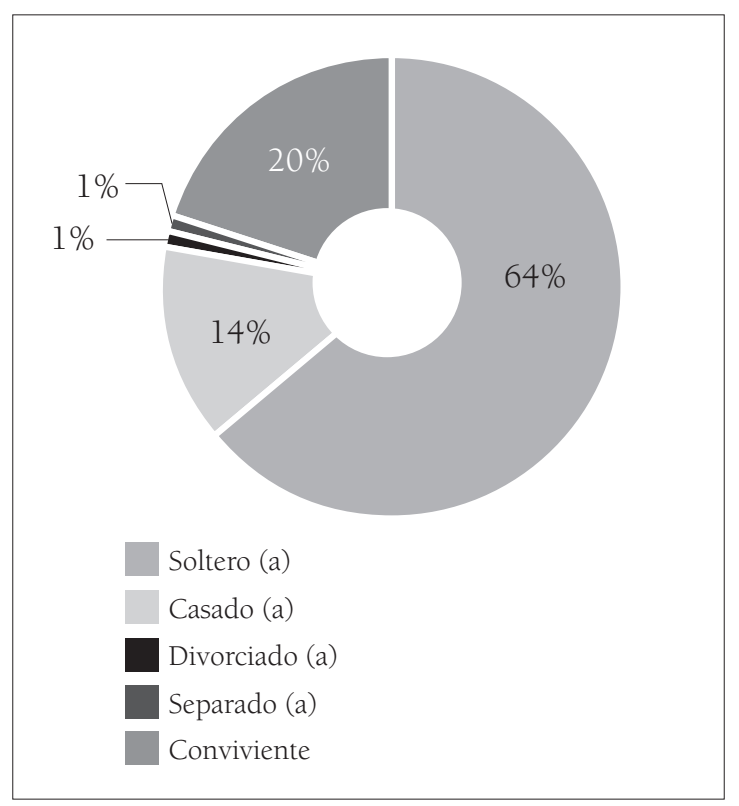

Fuente: Elaboración propia.

De 330 encuestados/as, 265 declara tener hijos/ as, lo que representa un $80,3 \%$ de los/as dominicanos/as participantes en este estudio. La mayor parte de encuestados/as que declaran tener hijos es mujer. Cuando se analiza este aspecto por zona de aplicación se observa que el 89,7\% (44) de los/as encuestados/as de Punta Arenas tienen hijos/as, a diferencia de los de la ciudad de Antofagasta, donde el 47,5\% declara no tenerlos, destacando por ser la ciudad donde los/as encuestados/as presentan menor presencia de hijos/as. Esto es importante de considerar a la hora de considerar las futuras reagrupaciones familiares.
FIGURA No 9: ENCUESTADOS/AS

CON O SIN HIJOS/AS (N: 330)

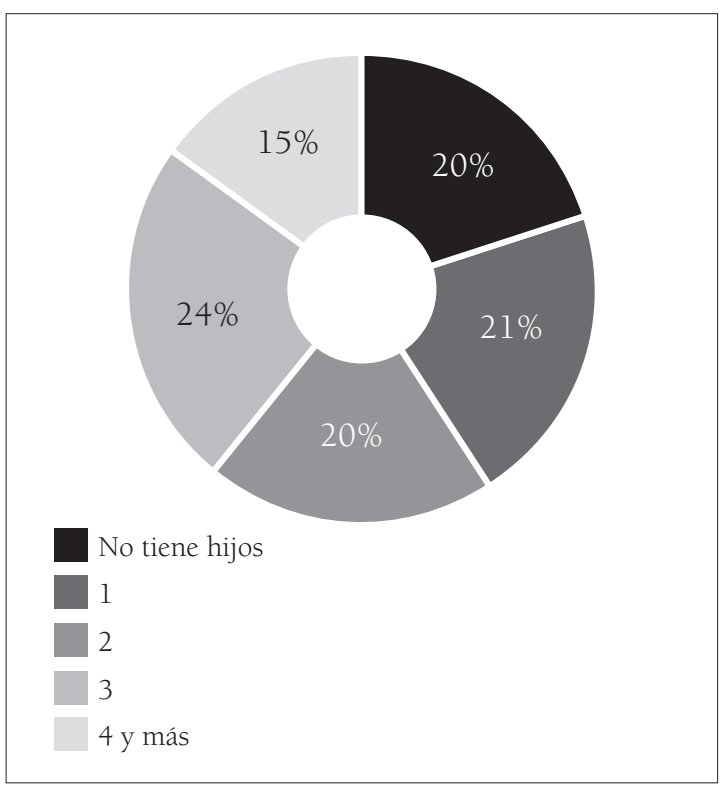

Fuente: Elaboración propia.

Cuando se analiza la edad de los/as hijos/as se observa que el 69,5\% de encuestados/as que afirma tener hijos/as son padres/madres de menores de 18 años. En promedio, la edad de los/as hijos/as de los participantes en este estudio es de 13 años, lo que da cuenta de la importancia de pensar políticas de migración que involucran a menores de edad.

FIGURA N ${ }^{\circ}$ 10: EDAD DE LOS HIJOS/AS ORDENADOS/AS POR TRAMOS (N: 235)

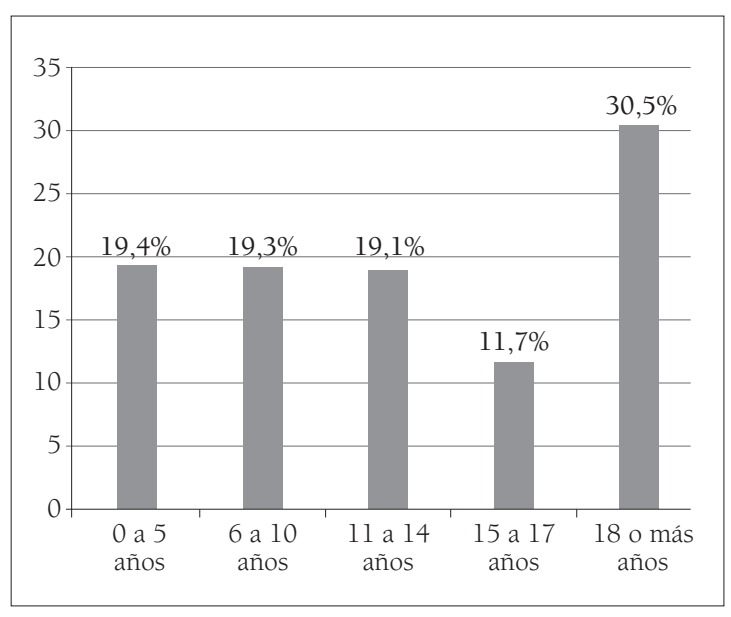

Fuente: Elaboración propia.

El gráfico anterior es especialmente relevante cuando se constata que la mayor parte de los/as encuestados/as que tienen hijos/as menores de 18 años 
señalan que sus hijos e hijas no viven con ellos/as, sino que se encuentran viviendo en República Dominicana o en otro país, al cuidado de otra persona. Sólo 49 de los/as 365 encuestados/as que declararon tener hijos/as afirman que éstos viven con ellos/ as en Chile, lo que corresponde a un 20,9\% de encuestados/as que respondieron a esta pregunta ${ }^{4}$.

Cuando se pregunta a los/as participantes en la encuesta acerca de los planes que tienen acerca de los hijos/as que no viven con ellos/as, se observa que el $44.9 \%$ de quienes responden a esta pregunta (83 dominicanos/as) espera a futuro traer a sus hijos/as al país, iniciando un proceso de reagrupación o reunificación familiar. Otro 10,8\% (20 dominicanos/ as) espera regresar a su país de origen para reunirse con éstos/as, mientras que el porcentaje restante aún no ha tomado una decisión al respecto o manifiesta un proyecto distinto.

\section{Remesas}

La mayor parte de los/as encuestados/as declara que envían dinero a sus familiares en República Dominicana (90,9\%). Más de la mitad (55\%) envía dinero a personas menores de 18 años que viven en su país de origen. El porcentaje restante envía dinero a personas mayores de 18 años, con quienes por lo general mantiene un vínculo de parentesco: padres, madres, hermanos/as e hijos/as, entre otros familiares directos. De hecho, los/as principales destinarios/as de las remesas que se envían a República Dominicana son hijos/as (79.5\%) o la madre (74.9\%) de los/as encuestados/as.

Lo habitual es que el envío de remesas se haga en forma periódica, mayoritariamente una vez al mes $(66,3 \%)$, aunque también se observan envíos quincenales o con frecuencias distintas según "necesidad". La mayor parte de encuestados/as considera que las remesas que ellos envían se usan preferentemente en alimentación $(60,4 \%)$, lo que explica su frecuencia y permanencia en el tiempo.

La mayor parte de encuestados/as afirma enviar un cuarto $(38,9 \%)$ o la mitad $(38,3 \%)$ de sus ingresos obtenidos en Chile a República Dominicana, tendencia que presenta algunas diferencias según zona de aplicación. Por ejemplo en Antofagasta predominan aquellos/as encuestados/as que envían un cuarto de sus ingresos (75\%) por sobre las otras categorías, mientras que en Iquique prevalece la tendencia mayoritaria de enviar la mitad del sueldo o más (48,6\%). Una mención aparte se presenta en el caso de 10 encuestadas de Punta Arenas, quienes declaran enviar prácticamente todo su sueldo a su país $(22,7 \%)$.

FIGURA N ${ }^{\circ}$ 11: PORCENTAJE DE INGRESOS QUE SE ENVÍA A REPÚBLICA DOMINICANA (N: 298)

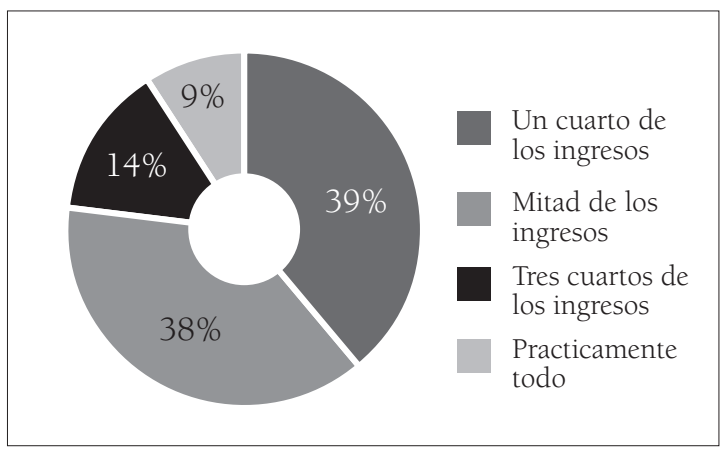

Fuente: Elaboración propia.

\section{Oportunidades y proceso de inclusión social}

Finalmente, los/as encuestados/as entregan su opinión (medida en una escala de liket) respecto de un conjunto de afirmaciones que muestran si Chile es un buen lugar para vivir: es así para el 59,7\% de los/ as encuestados/as, y reconocen que en el país han tenido oportunidades $(58,8 \%)$ que en otros lugares no tendrían. No obstante, cuando comparan las oportunidades que han tenido en el país con las que tiene una persona chilena, el 45,2\% (149) es enfático en afirmar que nunca o casi nunca se tienen las mismas oportunidades, situación que abre la posibilidad de profundizar en su proceso de integración al país y en algunas acciones de discriminación por parte de la comunidad receptora.

Esta afirmación se sustenta cuando se observa que el 46,9\% (155) de encuestados/as considera que nunca o casi nunca se respetan a las personas con independencia de su nacionalidad. La única excepción se encuentra en la zona de Antofagasta, donde el 70\% de encuestados/as de esa zona afirma que casi siempre se respetan a las personas con independencia de su nacionalidad.

4 Al respecto, es importante precisar que 30 encuestados/as no responden esta pregunta, principalmente porque no tienen hijos/as menores de edad, por lo que el análisis de la situación de cuidado de los/as hijos/as se hace sobre un número de 235 casos válidos. 
FIGURA N ${ }^{\circ}$ 12: RESPETO A LAS PERSONAS CON INDEPENDENCIA DE SU NACIONALIDAD (N: 330)

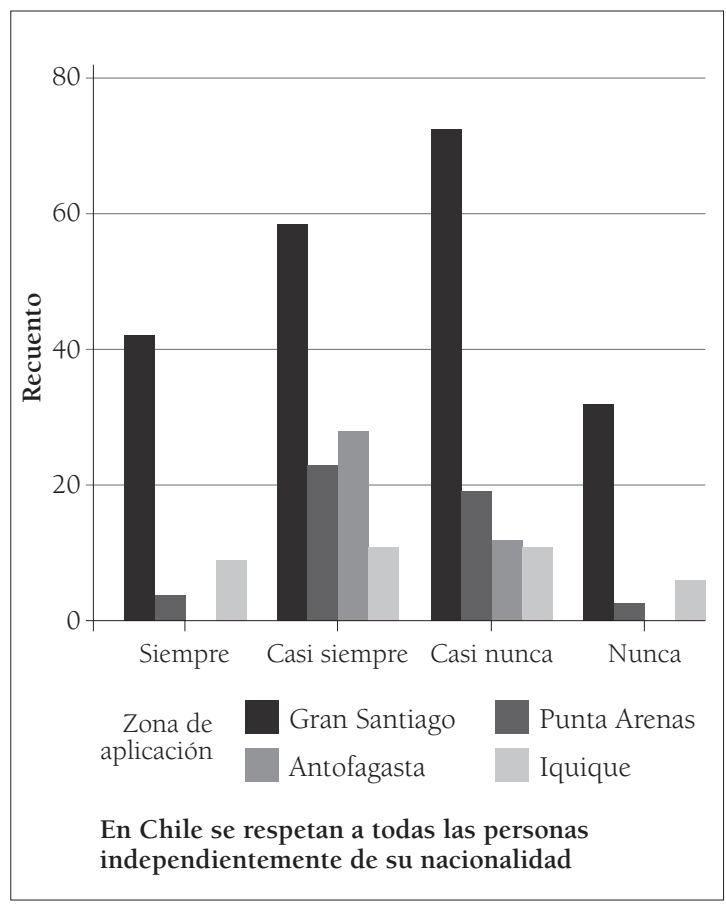

Fuente: Elaboración propia.

\section{Cierre conclusivo}

Como vemos, el proceso de instalación de la población dominicana que actualmente reside en Chile es diferenciado y tiene aspectos tanto positivos como negativos, que se deben considerar a la hora de gestionar los flujos migratorios provenientes desde este país y sus posibilidades efectivas de inclusión social y laboral. Desarrollar procesos de efectiva inclusión social de este colectivo implicará, por un lado, repensar las barreras de entrada que nublan su llegada al país -como el requisito de visa en el lugar de origen- hasta las lógicas de acogida que se desarrollan a nivel local para que este colectivo pueda gozar de las mismas oportunidades que cualquier otro/a ciudadano/a residente.

A través del estudio, pudimos visualizar que las personas dominicanas se enfrentan con una serie de obstaculizadores para su efectiva inclusión social, que no sólo devienen a partir de las aprensiones socioculturales y de un cierto rechazo en parte de la comunidad chilena de recepción, sino también a partir de dificultades impuestas desde el mismo Estado. Esto hace que su ubicación tanto residencial como laboral, social y económica tenga tramas diferenciadas de acuerdo al año de ingreso al país, como también dependiendo de las redes de contacto que poseen.
Por tanto, el problema administrativo y de regularidad sigue siendo un gran escollo para solventar una vida en igualdad de condiciones en un sector de esta población inmigrada. La visa de turismo que se exige en República Dominicana para ingresar a Chile emerge como el símbolo por excelencia que se enuncian respecto de las dificultades que deben cruzar. Luego, quienes no entran al país de forma regular se sitúan en una posición administrativa de difícil resolución a largo plazo. Así se presenta una suerte de círculo vicioso: aquellas personas dominicanas ubicadas en situación de vulnerabilidad quedan al margen de cualquier posibilidad de apoyo directo del Estado precisamente porque no disponen de los requisitos mínimos exigidos a nivel de visados. De esta manera, quienes cruzan una situación más frágil al estar en situación irregular son quienes menos posibilidades tienen de acceder a los beneficios públicos, la oferta programática del Estado y apoyos directos de los municipios. En ese sentido, las políticas públicas no están considerando como sujeto de derecho a personas dominicanas que entran por paso fronterizo no habilitado precisamente por no estar en situación irregular, o bien porque no llevan el tiempo suficiente en el país para acceder a ciertos programas públicos de apoyo.

Si bien se puede valorar los esfuerzos actuales de Chile -a través de medidas específicas de salud, vivienda, trabajo y educación recientemente incorporadas- en haber dado pasos hacia una inclusión en un sentido más amplio, no es posible por ahora asegurar que se han resuelto los nudos críticos por los que cruza la población dominicana debido al carácter complejo que comporta este tipo de inmigración.

Por otro lado, el hecho de que se trate de una población feminizada y de que la mayor cantidad de las personas haya dejado a sus hijos e hijas en su país de origen o en un tercer país, hace que la reunificación familiar sea un horizonte a tener presente, sobre todo con las expectativas en este sentido, que explicitan las personas entrevistadas de este estudio. Por tanto, pese a la incorporación en los últimos años de cierta retórica de "derechos" en las políticas, aún queda un largo camino por recorrer en la implementación de esta perspectiva. En definitiva, se requiere dar cuerpo al "enfoque de derechos", basado en principios y estándares internacionales y operacionalizarlo con tal de que estos derechos puedan ser respetados, protegidos y exigidos, con tal de sentar las bases de una buena inclusión social de la población dominicana. Queda aún mucho por hacer, pero se logra palpar cierta disposición políti- 
ca, en los esfuerzos desde diversos sectores sociales y estatales, y se vislumbran posibilidades en el horizonte.

\section{Bibliografía}

CAREF (2015). La Migración Dominicana en Argentina: Trayectorias en el Nuevo Siglo (2000-2015). Argentina: Ediciones Organización Internacional para las Migraciones (OIM) y Comisión Argentina para los Refugiados y Migrantes (CAREF).

DEM (2015). Anuario Estadístico nacional 2005-2014. Chile: Gobierno de Chile.

DEM (2016). Estadísticas Migratorias 2005-2015. Chile: Departamento de Extranjería y Migración, 2016. Recuperado el 19 de julio de 2016, de http://www.extranjeria.gob.cl/estadisticas-migratorias/

EMBAJADA DE REPÚBLICA DOMINICANA EN CHILE. (2016). Relaciones Bilaterales entre Chile y República Dominicana. Recuperado el 19 de julio de 2016, de http://www.embajadadominicana.cl/index.php/ gobierno/relaciones-bilaterales-entre-chile-y-republica-dominicana

ESPERÓN, F; DANTUR, Á. Y CARNERO, V. (2012). Seminario Migración, Género y Derechos. V Congreso de la Asociación Latinoamericana de Población. Montevideo, Uruguay. Recuperado el 14 de octubre de 2015, desde http://www.mininterior.gov.ar/asuntospoliticos/ pdf/seminario-migracion-genero-derechos.pdf

FERRER, M. (2006). La aplicación del enfoque de derechos humanos a los fenómenos de población: oportunidades y desafíos. Revista de Sociología, 20, 191-219.

GALAZ, C. (2009). Las relaciones de cooperación y exclusión entre personas con referentes diversos. Un estudio sobre la alteridad. Universidad Autónoma de Barcelona

GALAZ, C., FRÍAS, C. Y POBLETE, R. (2015). Inmigrantes en Chile: Oferta programática, participación, inclusión y vulnerabilidad. Chile: Ministerio de Desarrollo Social. ID.730566-23-LE14.Publicación restringida.

GAlAZ, C., POBlete, R Y FRÍAS (2017). Políticas Públicas e Inmigración. ¿Posibilidades de inclusión efectiva en Chile? Santiago: Editorial Universitaria.

GÓMEZ, I. (2014). Las Migraciones como Objeto de Interés de las Políticas Públicas: Un Enfoque de Derechos Humanos. En L. Burgogue-Larsen; A. Maués y B. Sánchez (Coords.). Derechos Humanos y Politicas Públicas. Manual (pp.235-273). España: Red de Derechos Humanos y Educación Superior (DHES).

GRAS, M. (2013). Desarrollo Local de Políticas Públicas con Enfoque de Derechos Humanos. VII Congreso Argentino de Administración Pública. Recuperado el 15 de octubre de 2015, de http://www.gobiernolocal.gob. ar/sites/default/files/Gras_Martin_Desarrollo_local_ de_politicas_publicas_con_enfoque_de_derechos_humanos_Panel_084.pdf

MARRERO, G. Y GUTIÉRREZ, I. (2009). Reflexiones sobre la emigración dominicana hacia España. En Anuario Digital CEMI: Migraciones Internacionales y Emigración Cubana (pp.199-234). Cuba: CEMI. Recuperado 20 de julio de 2016, desde http://bibliotecavirtual.clacso.org. ar/Cuba/cemi-uh/20110509044546/grian.pdf

MINISTERIO DE RELACIONES EXTERIORES, (2015). Chile y República Dominicana inician diálogo para negociar un acuerdo comercial. En prensa, 14 de enero de 2015. Recuperado el 19 de julio de 2016, desde https://www.direcon.gob.cl/2015/01/ chile-y-republica-dominicana-inician-dialogo-paranegociar-un-acuerdo-comercial-galeria-de-fotos/

MORAlES, A. (2007). La Diáspora de la Posguerra. Regionalismo de los migrantes y dinámicas territoriales en América Central. Colombia: FLACSO.

MUJICA, J. (2004) El desafío de la solidaridad: condiciones de vida y trabajo de los migrantes peruanos en Chile. Perú: OIT.

MUÑOZ, J. (2009). Derechos Humanos, Migraciones y Ciudadanía Universal. Diálogos Migrantes, 2, 8-20.

OCDE (2009). Notas de Países "República Dominicana". Recuperado el 28 de agosto de 2016, de https://www. oecd.org/dev/americas/45660833.pdf

PEÑA, J. (2012). Migraciones y Apertura Cosmopolita de la Ciudadanía. Arbor Ciencia, Pensamiento y Cultura, $188,755,529-542$.

PÉREZ DE LA FUENTE, Ó. (2006). Inclusión, Redistribución y Reconocimiento: Algunas Paradojas sobre los Inmigrantes. En I. Campoy (Coord.). Una discusión sobre la universalidad de los Derechos Humanos y la inmigración (pp.239-270). España: Dykinson.

SANTAMARÍA, E. (2002). La incógnita del extraño. Una aproximación a la significación sociológica de la "inmigración no comunitaria". España: Anthropos.

TIJOUX, M. E. Y RETAMALES, H. S. (2015). Trayectorias Laborales de Inmigrantes Peruanos en Santiago: El origen de excepción y la persistencia del "lugar aparte". Latin American Research Review, 50, 1, 135-153.

USHER, E. (2004). Fundamentos de Gestión de la Migración. Para las Personas Encargadas de Formular Politicas y Profesionales. Suiza: Organización Internacional para las Migraciones.

VALLENAS, K. (2011). El enfoque de Derechos Humanos en la Gestión de los Gobiernos Locales. Perú: Instituto de Estudios Peruanos.

YUFRA, L. (2013). Inmigrantes en formación: Estado de bienestar y promoción de lazos sociales en el caso de la formación para la inserción laboral de las personas inmigrantes en Barcelona. Athenea Digital, 13, 2, 301 209. 